\title{
Quantification of Swelling Characteristics of Pharmaceutical Particles
}

\author{
Mithushan Soundaranathan ${ }^{\mathrm{a}, \mathrm{b}}$, Pattavet Vivattanaseth ${ }^{\mathrm{a}, \mathrm{b}, \mathrm{e}}$, Erin Walsh $^{\mathrm{a}, \mathrm{b}}$, Kendal \\ Pitt $^{\mathrm{c}}$, Blair Johnston ${ }^{\mathrm{a}, \mathrm{b}, \mathrm{d}}$, Daniel Markl ${ }^{\mathrm{a}, \mathrm{b}, *}$ \\ ${ }^{a}$ Strathclyde Institute of Pharmacy and Biomedical Sciences, University of Strathclyde, Glasgow, G4 \\ ORE, UK \\ ${ }^{b}$ Future Continuous Manufacturing and Advanced Crystallisation Research Hub, University of \\ Strathclyde, Glasgow, G1 1RD, UK \\ ${ }^{c}$ Pharma Supply Chain, GlaxoSmithKline, Ware, SG12 ODE, UK \\ ${ }^{d}$ National Physical Laboratory, Teddington, TW11 OLW, UK \\ ${ }^{e}$ School of Pharmaceutical Sciences, University of Phayao, Phayao, Thailand
}

\begin{abstract}
Particle swelling is a crucial component in the disintegration of a pharmaceutical tablet. The swelling of particles in a tablet creates stress inside the tablet and thereby pushes apart adjoining particles, eventually causing the tablet to break-up. This work focused on quantifying the swelling of single particles to identify the swelling-limited mechanisms in a particle, i.e. diffusion- or absorption capacity-limited. This was studied for three different disintegrants (sodium starch glycolate/SSG, croscarmellose sodium/CCS, and low-substituted hydroxypropyl cellulose/L-HPC) and five grades of microcrystalline cellulose (MCC) using an optical microscope coupled with a bespoke flow cell and utilising a single particle swelling model. Fundamental swelling characteristics, such as diffusion coefficient, maximum liquid absorption ratio and swelling capacity (maximum swelling of a particle) were determined for each material. The results clearly highlighted the different swelling behaviour for the various materials, where CCS has the highest diffusion coefficient with $739.70 \mu \mathrm{m}^{2} / \mathrm{s}$ and SSG has the highest maximum absorption ratio of $10.04 \mathrm{~g} / \mathrm{g}$. For the disintegrants, the swelling performance of SSG is diffusion-limited, whereas it is absorption capacity-limited for CCS. L-HPC is both diffusion- and absorption capacity-limited. This work also reveals an anisotropic, particle facet dependant, swelling behaviour, which is particularly strong for the liquid uptake ability of two MCC grades (PH101 and PH102) and for the absorption capacity of CCS. Having a better understanding of swelling characteristics of single particles will contribute to improving the rational design of a formulation for oral solid dosage forms.
\end{abstract}




\section{Graphical Abstract}

\section{Quantification of Swelling Characteristics of Pharmaceutical Particles}

Mithushan Soundaranathan, Pattavet Vivattanaseth, Erin Walsh, Kendal Pitt, Blair Johnston, Daniel Markl

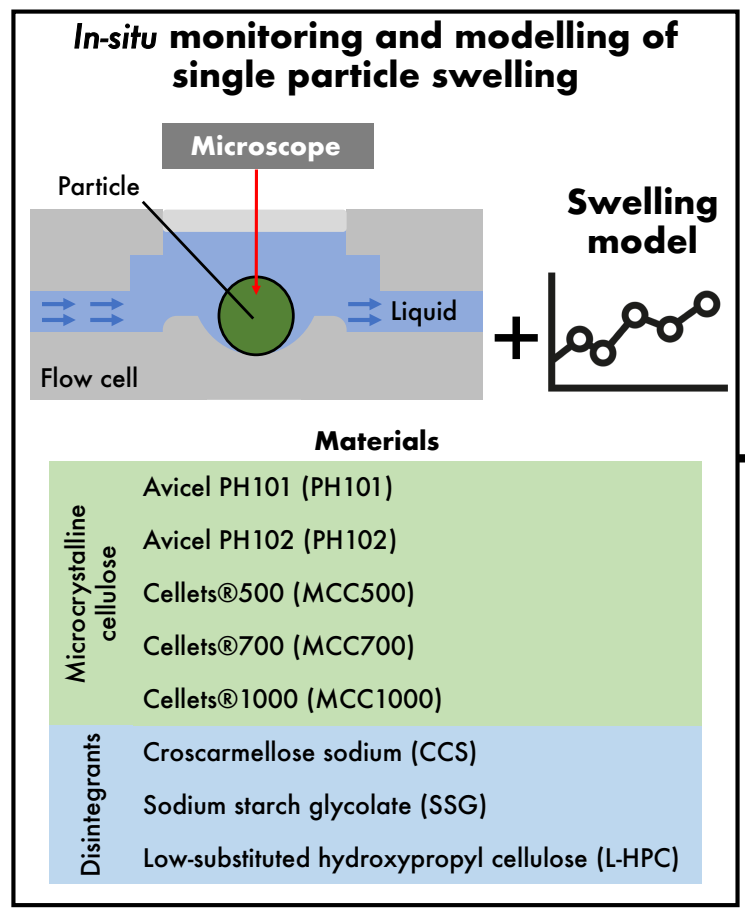

Characteristic swelling properties and swelling-limiting mechanisms

Characteristic swelling properties

- Maximum swelling capacity ( $\mu \mathrm{m})$

- Diffusion coefficient $\left(\mu \mathrm{m}^{2} / \mathrm{s}\right)$

- Maximum absorption ratio $(\mathrm{g} / \mathrm{g})$

Limiting mechanisms of disintegrants

- Diffusion-limited: SSG and L-HPC

- Absorption capacity-limited: CCS and L-HPC

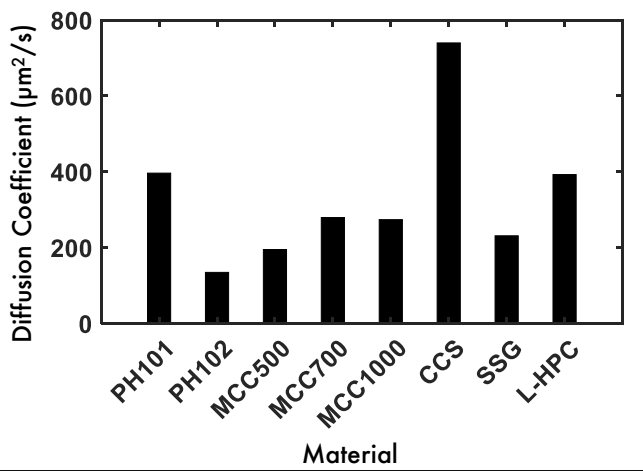




\section{Highlights}

\section{Quantification of Swelling Characteristics of Pharmaceutical Particles}

Mithushan Soundaranathan, Pattavet Vivattanaseth, Erin Walsh, Kendal Pitt, Blair Johnston, Daniel Markl

- In-situ monitoring of single particle swelling of eight different pharmaceutical materials.

- Extraction of characteristic swelling properties from the in-situ data coupled with a swelling model.

- Classification of materials into liquid uptake ability-limited and absorption capacity-limited swelling.

- Revealing anisotropic, particle facet depending, swelling behaviour of most materials. 
Keywords: Pharmaceutical, Disintegration, Particle, Swelling, Anisotropy, Diffusion

\section{Introduction}

Once a tablet administered orally comes in contact with a physiological fluid, the liquid will penetrate the porous tablet and in the majority of cases initiate its swelling. This swelling builds up an internal stress that causes the break up of the tablet into smaller agglomerates and the primary particles. These steps are referred to as the tablet disintegration processes, which are critical steps to dissolve and enable the absorption of the drug substance (Markl and Zeitler, 2017; York, 2018). A controlled disintegration process is essential to ensure the desired on-set of the therapeutic effect.

Tablet swelling is the most accepted mechanism for tablet disintegration (Patel and Hopponent, 1966) and it primarily depends on the swelling of the individual particles. To be able to disintegrate, the interparticulate bonds inside a tablet need to be disrupted by the swelling of excipient particles (Quodbach and Kleinebudde, 2015). Particle swelling is the volumetric and omnidirectional expansion of the particle during liquid contact (Faroongsarng and Peck, 1994). The swelling ability of the particle depends on the particle size, chemical structure and degree of crosslinking of polymeric systems (Desai et al., 2015; Bell and Peppas, 1996). To prevent polymers from dissolving in the aqueous media during swelling, other polymers are used to bind them, which are referred to as cross-linkers (Sweijen et al., 2017a). It has been observed that polymers with cross-linking produce a high swelling force but have a limited volume expansion, however, the disintegration time is quicker compared to other strongly swelling particles (Quodbach and Kleinebudde, 2015). Typically, disintegrants are added to a formulation as a swelling agent, and therefore enhance the disintegration process.

Common disintegrants include starch- and cellulose-based excipients such as corn starch, partially pregelatinized starch and lowsubstituted hydroxypropyl cellulose (L-HPC) (Patel and Hopponent, 1966; Desai et al., 2015; Quodbach and Kleinebudde, 2015). To accelerate the disintegration process further, synthetic polymers such as sodium starch glycolate (SSG), croscarmellose sodium (CCS) and crospovidone (XPVP) were developed, which are referred to as superdisintegrants. Besides the swelling of disintegrants, other excipients such as microcrystalline cellulose

\footnotetext{
${ }^{*}$ Corresponding Author: daniel.markl@strath.ac.uk
} 
(MCC)- typically used as a diluent, filler or binder - may also exhibit swelling and contribute to the overall swelling of a tablet (Reier and Shangraw, 1966; Desai et al., 2015).

Generally, materials have been classified in either omni-directional or unidirectional (against the direction of compaction) swelling. The uni-directional swelling is commonly referred to as strain recovery, which is the reversible viscoelastic process of deformation. The particles recover to their original shape by the mechanical activation of the disintegrant polymer when the particle comes in contact with liquid. The polymer chain then adopts to the most energy-favourable position and causes a uni-direction expansion of the particle (Desai et al., 2015). Crospovidone is considered as a material that undergoes strain recovery (Desai et al., 2012; Quodbach and Kleinebudde, 2014; Berardi et al., 2018). The accepted mechanism of MCC, L-HPC, CCS and SSG is omni-directional swelling, which is driven by the swelling ability of the particles. For omni-directional swelling materials the swelling ability is a material property, whereas for strain recovery materials it is dominated by the manufacturing settings (e.g. compression force in tableting).

A range of studies have been performed to quantify the swelling of particles and also to model the swelling. The swelling of disintegrants in a suspension was quantified by Zhao and Augsburger (2005) using laser diffraction. They measured the volume mean diameter change during swelling and revealed that SSG had better swelling capacity (maximum swelling of particle) than CCS. Rudnic et al. (1982) analysed the wetting and swelling of individual SSG and sodium carboxymethyl stach particles using an optical microscope. Their results showed that the rate and extent of swelling for the measured particle varied with particle size, i.e. larger particles showed substantially greater rates and extent of swelling compared to smaller particles.

Rojas et al. (2012) quantified the swelling value - the ratio between powder expanded volume upon water absorption and the initial sample weight - and water uptake ability of SSG, CCS and MCC powder in simulated gastric and intestinal fluid. SSG had the largest water uptake ability and swelling value followed by CCS and MCC. Desai et al. (2012) analysed and quantified the swelling of compacted disintegrant particle that contained $70 \%$ disintegrant and $30 \%$ glass beads using an optical microscope and a high speed camera. Their results revealed the trend in cross-sectional area increase during swelling for various compacts was as follows: SSG, CCS, MCC and L-HPC.

Berardi et al. (2018) analysed the disintegration mechanism and quantified the swelling of pure CCS, SSG and XPVP tablets. SSG tablets swelled faster and more extensively compared to CCS tablets. The authors described the swelling mecha- 
nism of XPVP as strain recovery (swelling in axial direction). Botzolakisi and Augsburger (1988) quantified the swelling and liquid uptake rate of pure disintegrant tablets revealing that CCS tablets exhibited the greatest swelling and highest liquid uptake rate followed by SSG. Several studies quantified the disintegrant swelling of powder compacts Rojas et al. (2012); Desai et al. (2012); Berardi et al. (2018); Botzolakisi and Augsburger (1988) and suspensions (Zhao and Augsburger, 2005). The swelling behaviour of a group of particles (e.g. powder bulk or compacts) is not only influenced by the single particle swelling, but also by the microstructure of the entire sample. The microstructure of a bulk of particles - loose particles or as a compact - strongly influences the wetting process of the particles (Markl et al., 2018b). In such a case, the particles are wetted by a liquid front that moves from the surface through the sample. The total swelling of the bulk of particles is thus a superposition of asynchronous swelling of individual particles. The swelling of a bulk of particles is directly controlled by the wetting process and hence strongly depends on additional factors such as the used preparation method as well as particle properties (e.g. size, shape, surface energy) (Al-Sharabi et al., 2020). Although the swelling behaviours of a bulk of particles can be compared between different materials by ensuring that microstructural factors are negligible, the extracted swelling characteristics cannot be generalised. Characteristic swelling properties should be independent of the bulk behaviour in order to make it generally applicable and for it to be useful for a rational design of a formulation and the manufacturing conditions. This study aims to determine such characteristic swelling properties for common pharmaceutical excipients. The analysis of single non-pharmaceutical particle swelling is commonly performed using an optical microscope (Sweijen et al., 2017a; Gasmi et al., 2015; Esteves, 2011). An environmental scanning electron microscope (ESEM) has also been applied to analyse the swelling of single particles (Jenkins and Donald, 1997).

To better understand the swelling behaviour, several groups have developed models to describe the swelling of individual particles as a function of time. These models range from (semi-)empirical (Omidian et al., 1998; Esteves, 2011) to physical based models (Sweijen et al., 2017a,b). Sweijen et al. (2017a) developed a swelling rate equation considering the diffusion of water into a single spherical super absorbent polymer (SAP) particle. The same group improved their model for SAP particles to account for an irregular particle shape, water uptake on the surface, diffusion into the particle and subsequence particle swelling Sweijen et al. (2017b). Markl et al. (2017b) modified a mathematical model based on an empirical equations from Schott (1992) for MCC particles to describe the tablet swelling and also the liquid penetration kinetics. Kimber et al. (2012) developed a model for swelling of polymer particles considering Fickian mass transfer, which was incorporated in a 
discrete element model to simulate tablet swelling and dissolution.

This study presents a method for determining anisotropic swelling descriptors of single pharmaceutical particles by combining in-situ measurements with a swelling model. A bespoke flow cell coupled to an optical microscope was developed to monitor single particle swelling in real-time. The particle swelling was measured from the microscope images using a bespoke algorithm. From the collected swelling data, the diffusion coefficient, maximum absorption and swelling capacity of the materials were determined. This study was conducted for the most common superdisintegrants (CCS, L-HPC and SSG) that are classified as omni-directional swelling materials and also for five different grades of MCC.

\section{Material and Methods}

\subsection{Materials}

This study was conducted for five different grades of MCC and three different superdisintegrants: Cellets ${ }^{\circledR} 500$ (MCC500, Harke Pharma, Dresden, Germany), Cellets ${ }^{\circledR} 700$ (MCC700, Harke Pharma, Dresden, Germany) and Cellets®1000 (MCC1000, Harke Pharma, Dresden, Germany) and MCC PH101 (Avicel PH101, Roquette, Lestrem, France), MCC PH102 (Avicel PH102, FMC International, Philadelphia, USA) as well as sodium starch glycolate (Primojel®, SSG, DFE Pharma, Goch, Germany), croscarmellose sodium (Ac-Di-Sol, CCS, SDW-802, FMC International, Philadelphia, USA) and low-substituted hydroxypropyl cellulose (LHPC, Shin Etsu, Tokyo, Japan).

The MCC grades differ in terms of particle size, microstrucutre and moisture content. MCC is produced through an acid hydrolysis process by spray drying, which breaks down only the amorphous region of the cellulose. Cellulose consists of several $\beta 1-4$-linked glucose subunits and is more crystalline than starches. Cellulose consist of both a compact microcrystalline region and less dense amorphous region (Thoorens et al., 2014; Desai et al., 2015). PH101 and PH102 are produced by varying and controlling the spray drying conditions, and thereby control the agglomeration (particle size distribution) and moisture content (loss on drying) (Thoorens et al., 2014). The larger MCC grades (MCC500-1000) are made from MCC powder (Russell et al., 2018) through direct wet pelletisation or extrusion-spheronisation (Kleinebudde and Knop, 2007) in order to manufacture highly spherical particles.

\subsection{Particle Characterisations}

\subsubsection{Particle Size}

The dynamic imaging instrument QICPIC (Sympatec GmbH, ClausthalZellerfeld, Germany) was used to characterise the particles in terms of size and 
shape. Before the measurement approximately $2 \mathrm{~g}$ of the material was dispersed into the measurement area with a speed of up to $100 \mathrm{~m} / \mathrm{s}$ using the RODOS powder disperser. The M7 lens (size range 4.2 - $8665 \mu \mathrm{m}$ ) was selected for this study, and three replicates were performed for each material to determine the average particle size $\left(D_{50}\right)$ and sphericity $\left(S_{50}\right)$. The particle size represents the equivalent circle diameter. The sphericity is the ratio between the perimeter of a circle with the same area as the particle and the real perimeter. $S_{50}$ is in the range of 0 and 1 . The smaller the value, the more irregular the shape of the particle is.

\subsubsection{Particle Density Measurements}

The true density of the materials were measured by helium pycnometer ( $\mathrm{Mi}$ croUltrapyc 1200, Quantachrome instrument, Graz, Austria). The test was carried out using a multi-run system (three runs) with a sample size of $1 \mathrm{~g}, 0.5 \mathrm{~g}$ and $0.1 \mathrm{~g}$ for MCC500-1000, PH101/PH102 and the disintegrants, respectively.

\subsubsection{Maximum absorption ratio measurement}

The maximum absorption ratio of single particles $\left(Q^{\max }\right)$ was estimating by placing $0.5 \pm 0.1 \mathrm{~g}$ for MCC500-1000 and $0.7 \pm 0.1 \mathrm{~g}$ PH101/PH102, of dry material in a beaker and subsequently hydrating it. After 40 min the water was filtrated by vacuum filtration, and the mass of the swollen particles were measured. $Q^{\max }$ is the ratio between the mass of the hydrated particles and the mass of the dry particle.

\subsection{In-situ Measurement of Swelling of Single Particles}

\subsubsection{Custom-built flow cell for swelling measurements}

A flow cell was developed to in-situ monitor the swelling of single particles. The main requirements for the flow cell were:

1. have an enclosed space for the particle, where it can be monitored with a microscope.

2. prevent particles from moving.

3. wet the entire particle instantaneously.

4. have a continuous flow to control the environment (liquid properties).

If the particle is not wetted entirely and instantaneously, then we would need to account for the contact angle and the wetted surface in the swelling models. The contact angle, however, cannot be determined accurately for a single (and probably porous) particle.

The design of the flow cell is given in Figure 1. The flow cell was printed using a Formlabs Stereolithography (SLA) 3D printer with a clear V4 resin. After printing 

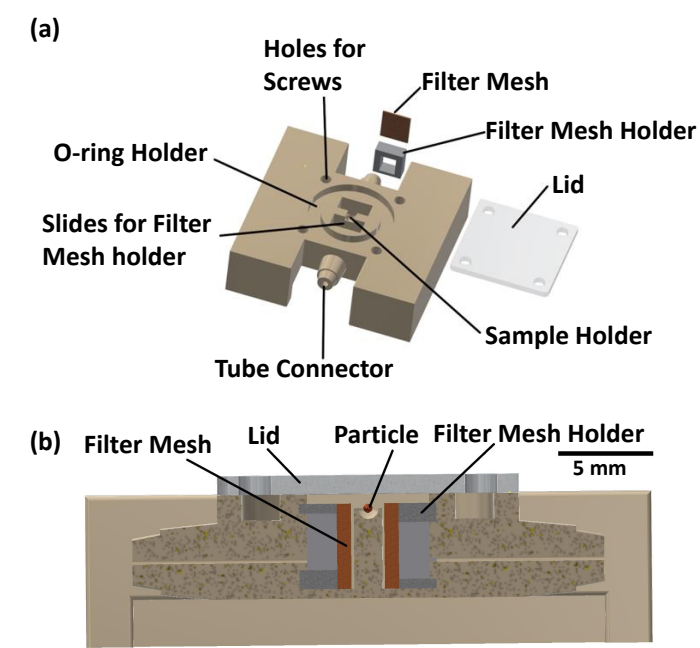

Figure 1: Schematic representation of the flow cell. (a) An explosion view of the 3D design of the flow cell. (b) Cross-section view of the flow cell indicating the particle position.

the lid, the lid was sanded to increase the optical transparency. Which allowed seeing through the lid. To close the lid, a $17 \mathrm{~mm}$ long screw and nuts with $2 \mathrm{~mm}$ outer diameter (O.D). were used.

The particle is placed in a spherical-shaped sample holder with a diameter of $3 \mathrm{~mm}$ and a total volume of $0.03 \mathrm{~mL}$. Tube connectors for inlet and outlet are designed into the flow cell. A filter mesh was placed after the inlet and before the outlet to prevent the particle moving into the tubes and leaving the area of view of the microscope. A twill woven wire mesh with a $0.026 \mathrm{~mm}$ aperture and $0.025 \mathrm{~mm}$ wire diameter from The Mesh Company (Warrington) Ltd was used for this purpose.

The flow cell was sealed by using an RS PRO nitrile rubber O-ring. The O-ring has an inner diameter (I.D). of $18.66 \mathrm{~mm}$, O.D. of $25.72 \mathrm{~mm}$ with a thickness of $3.18 \mathrm{~mm}$. The total height of the setup is $18 \mathrm{~mm}$ including the $2 \mathrm{~mm}$ thick lid.

\subsubsection{Experimental Setup}

Figure 2 illustrates a schematic representation of the experimental setup of the flow cell coupled with an optical microscope to measure the swelling of a single particle.

Deionised water at $20^{\circ} \mathrm{C}$ was pumped from a $500 \mathrm{~mL}$ glass bottle into the custombuilt flow cell by a peristaltic pump (520s, Watson Marlow Ltd) with a flow rate of $1.83 \mathrm{~mL} / \mathrm{min}$ through tubes with an O.D. of $6 \mathrm{~mm}$ and an I.D. of $2 \mathrm{~mm}$. The swelling process was monitored by an optical microscope (Leica DM6000, Leica Microsystems CMS GmbH, Germany) with a magnification of 10x for PH101 and PH102, 5x for 


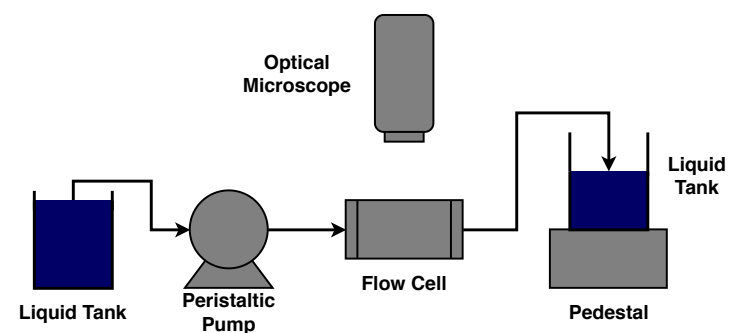

Figure 2: Schematic representation of the experimental setup of the flow cell coupled with an optical microscope.

MCC500-1000, 5x/10x for CCS, 10x for SSG and 10x/20x for L-HPC. The frame rate of the videos capturing of the swelling process was $10 \mathrm{fps}$. The outlet glass container is placed on a pedestal to generate a pressure difference between the flow cell and the outlet container to avoid the formation of bubbles inside the flow cell during the measurement as they may affect the swelling measurement. Six individual particles were measured per material.

\subsection{Data Analysis to Quantify Single Particle Swelling}

Individual frames from the optical microscope were processed to quantify the swelling of the particles. In this study, the processing techniques applied on the microscopic images are grayscale conversion, denoising and binarisation by thresholding. The entire workflow to analyse and quantify the swelling of a single particle is presented in Figure 3.

Image denoising is used to remove random fluctuations in pixels values which arise from the characteristics of the image acquisition (Russ and Neal, 2016). There are many sources of noise in images and one of the major reasons for noise in the microscope images of this study is the variation of the brightness. The image sensors count photons, the counting process is randomly quantified, and therefore the microscope images also often have photon counting noise (Boncelet, 2009). In this study, an edge preserving filter, i.e. an anisotropic diffusion filter (Perona and Malik, 1990; Manjón et al., 2008), was applied for denoising the individual images. The edges are preserved by averaging pixels in the orthogonal direction of the local gradient (Manjón et al., 2008). The binarisation of the denoised microscope images was performed by two different methods depending on the particle size. If the particle diameter was larger than $250 \mu \mathrm{m}$, the image was binarised by thresholding. If particle diameter was smaller $250 \mu \mathrm{m}$, the particle shape was first detected using the underlying edge and then the enclosed object was binarised.

The edge and centroid of the particle was detected, and the distance from centroid to each point on the edge was determined. The particle radius $\left(r_{P}\right)$ was calcu- 


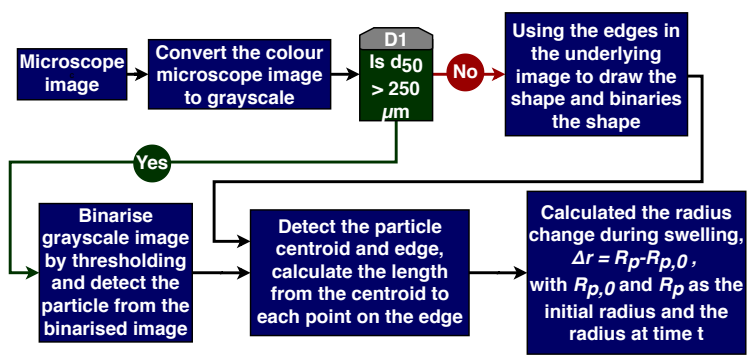

Figure 3: The workflow of the data processing to analyse and quantify the swelling of single particles.

lated from an average distance from the centroid to the edge points of the particle. The object properties (area, perimeter, minor axis and major axis of a fitted ellipsoid) were calculated. The analysis was implemented in Matlab (2019b, Mathworks, Massachusetts, USA)

\subsection{Swelling Model}

The swelling model in this study to extract characteristic swelling properties is based on the model from Sweijen et al. (2017a), which was originally developed for describing the swelling process of SAP particles. The absorption ratio $\left(Q_{i}^{\text {abs }}\right)$ of each individual particle $i$ is described by the mass of absorbed water $\left(m_{i}^{w}\right)$ and the dry mass of the particle $\left(m_{i}^{s}\right)$ :

$$
Q_{i}^{\mathrm{abs}}=\frac{m_{i}^{w}+m_{i}^{s}}{m_{i}^{s}}=\frac{r_{p}^{3} \rho_{w}}{r_{p, 0}^{3} \varrho_{s}}-\frac{\varrho_{w}}{\varrho_{s}}+1 .
$$

$r_{p, 0}$ is the initial particle radius, $r_{p}$ is the particle radius at time $t, \varrho_{s}$ is the density of a dry particle and $\varrho_{w}$ is the density of the liquid (deionised water in this study).

The swelling of a particle is driven by the difference in chemical potential between the particle and water (liquid medium) Huyghe and Janssen (1997). Therefore, it is assumed that the swelling is attributed to the diffusion of the liquid into the particle. The absorption rate can thus be described by (Sweijen et al., 2017a)

$$
\begin{aligned}
\frac{d Q_{i}^{\mathrm{abs}}}{d t} & =K_{i}\left(\frac{Q^{\mathrm{max}}-Q_{i}^{\mathrm{abs}}}{Q_{i}^{\mathrm{abs}}}\right), \\
K_{i} & =\frac{3 D r_{p}}{r_{p, 0}^{3}} .
\end{aligned}
$$




\begin{tabular}{ccccc}
\hline Material & $D_{50}(\mu \mathrm{m})$ & $S_{50}$ & $\varrho_{s}\left(\mathrm{~g} / \mathrm{cm}^{3}\right)$ & $Q^{\max }(\mathrm{g} / \mathrm{g})$ \\
\hline PH101 & $78 \pm 1$ & $0.73 \pm 0.00$ & $1.561 \pm 0.003$ & $1.45 \pm 0.31$ \\
PH102 & $111 \pm 1$ & $0.75 \pm 0.01$ & $1.564 \pm 0.008$ & $1.38 \pm 0.15$ \\
MCC500 & $662 \pm 2$ & $0.94 \pm 0.00$ & $1.441 \pm 0.002$ & $1.19 \pm 0.06$ \\
MCC700 & $924 \pm 6$ & $0.94 \pm 0.00$ & $1.446 \pm 0.002$ & $1.20 \pm 0.03$ \\
MCC1000 & $1215 \pm 20$ & $0.94 \pm 0.00$ & $1.437 \pm 0.004$ & $1.18 \pm 0.02$ \\
CCS & $54 \pm 1$ & $0.66 \pm 0.01$ & $1.403 \pm 0.017$ & - \\
SSG & $54 \pm 1$ & $0.87 \pm 0.02$ & $1.414 \pm 0.001$ & - \\
L-HPC & $79 \pm 0$ & $0.63 \pm 0.00$ & $1.136 \pm 0.095$ & - \\
\hline
\end{tabular}

Table 1: Size, shape, density and maximum absorption ratio of the particles.

$D\left(\mu \mathrm{m}^{2} / \mathrm{s}\right)$ is the diffusion coefficient for water molecules in the particle, which is assumed to be constant. $Q^{\max }$ is the maximum absorption ratio. For spherical particles and an incompressible liquid, Eq. 2 can be rewritten in term of $\frac{d r_{p}}{d t}$ as function of $r_{p}$ :

$$
\frac{d r_{p}}{d t}=\frac{D \varrho_{s}}{r_{p} \varrho_{w}}\left(\frac{Q^{\mathrm{max}}-Q_{i}^{\mathrm{abs}}}{Q_{i}^{\mathrm{abs}}}\right) .
$$

\section{Results and Discussion}

\subsection{Particle Characterisation}

Table 1 shows the results from the particle characterisation including the particle size, sphericity, true density and maximum absorption ratio of the different materials. Unsurprisingly, MCC1000 has the largest particle size, while SSG and CCS have the smallest. The large MCC grades (MCC500-1000) are almost spherical $\left(S_{50}>0.9\right)$ and SSG particles are the most spherical of the disintegrants. CCS and L-HPC have a needle-like shape, which is in agreement with the literature (Desai et al., 2015). MCC PH101 and PH102 have a similar cylindrical shape (Desai et al., 2015).

$Q^{\text {max }}$ could only be measured for the different MCC grades as SSG formed a gel and CCS/L-HPC dissolved during the measurement. MCC500-1000 have very similar $Q^{\max }$ values and the relative difference between PH101 and PH102 is only 5\%. Since the larger MCC grades (MCC500-1000) are made from MCC powder (Russell et al., 2018) through direct wet pelletisation or extrusion-spheronisation (Kleinebudde and Knop, 2007), a lack of difference in terms of $Q^{\text {max }}$ between the 


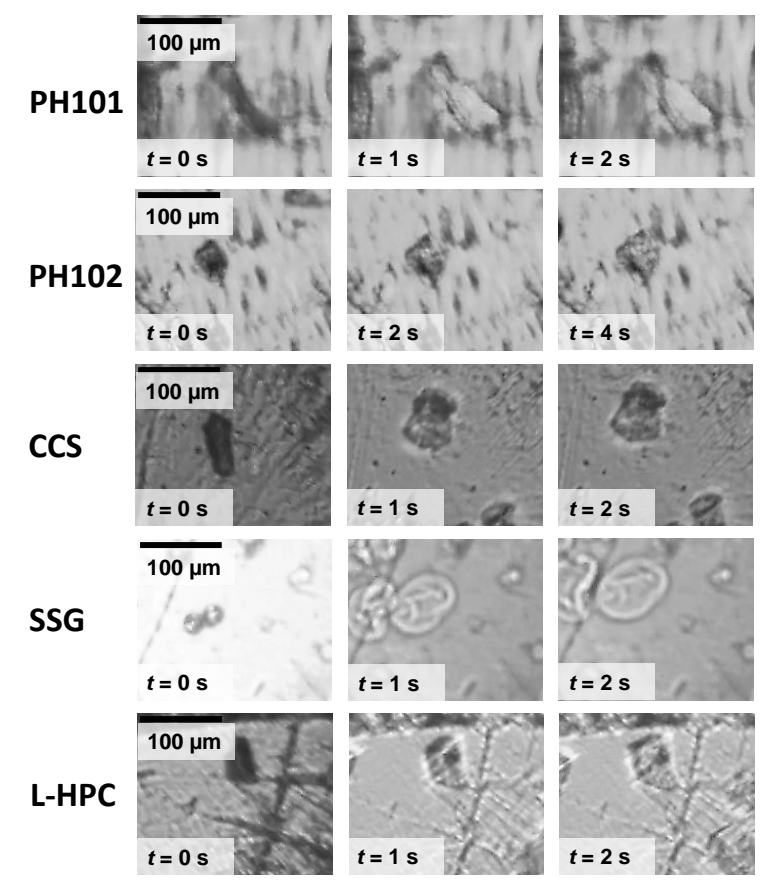

Figure 4: Microscope images of the disintegrant and PH101/PH102 particles from different time points during swelling. The time points were 0,1 and $2 \mathrm{~s}$ for all materials except for PH102 where images were taken at 0,2 and $4 \mathrm{~s}$ due to its slower swelling behaviour.

smaller and larger MCC grades is not surprising. The results also indicate that the $Q^{\max }$ of the particle is primarily material dependent and is not affected by particle size.

\subsection{Swelling of Single Particle}

\subsubsection{Swelling Data}

Figures 4 and 5 depict the images of a particle at different time points during the swelling process for the different materials. These images were processed (see Figure 3) to extract the change of radius as a function of time. The measured particle radius change, $\Delta r$, during swelling of different materials are given in Figure 6 , which clearly indicate a varying swelling rate and also swelling capacity $\left(\Delta r^{\max }\right)$, i.e. the maximum swelling of a particle.

The swelling capacity of the MCC particle indicates to be size dependent, since the larger MCC1000 particle showed the greatest swelling capacity followed by MCC700, MCC500, PH101 and PH102. $D_{50}$ values (Table 1) shows that PH102 


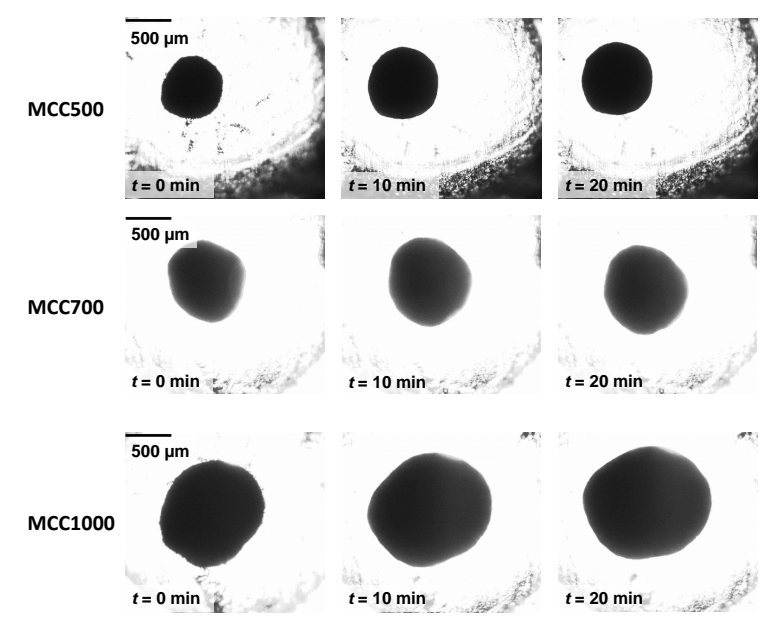

Figure 5: Microscope images of the MCC500-1000 particles from different time points during swelling. The time points were 0,10 and 20 min for all materials.

is larger than PH101 leading to different swelling profiles where PH101 swells 5 $\mu m$ within $3 \mathrm{~s}$ and PH102 swells $4 \mu m$ within $6 \mathrm{~s}$ (Figure 6a).

Both L-HPC and CCS are modified cellulose with a significantly larger swelling capacity than MCC (Figure 6 ). L-HPC is a modified hydrophilic, water-insoluble cellulose (a low substituted form of cellulose ether), whereas CCS is a crosslinked carboxymethyl cellulose sodium (Desai et al., 2015). The results indicate that SSG has the highest swelling capacity followed by CCS and L-HPC. This is in agreement with the results from Zhao and Augsburger (2005); Desai et al. (2012). The results from Zhao and Augsburger (2005) showed that volume mean diameter change during swelling of SSG was greater than CCS. This is also in agreement with Desai et al. (2012) who demonstrated that the swelling of SSG compacts is greater than that of CCS compacts followed by L-HPC compacts. SSG is the sodium salt of crosslinked carboxymethylated starch, modified by two chemical processes: substitution to increase hydrophilicity and cross-linking to reduce solubility (Desai et al., 2015; Shah and Augsburger, 2002). The high swelling capacity of SSG is due to the high spacing between cross-linking of the phosphate groups. The high spacing allows water penetration and swelling and facilitates gel formation (Rojas et al., 2012). In contrast, the CCS cross-linking through esterification does not allow high spacing between polymer chain (Zarmpi et al., 2017) and thus has a lower swelling capacity as seen in Figure 6c.

The particle swelling process consist of two mechanisms: water uptake at the particle surface and the swelling ability of the particle. Since SSG has a relatively 

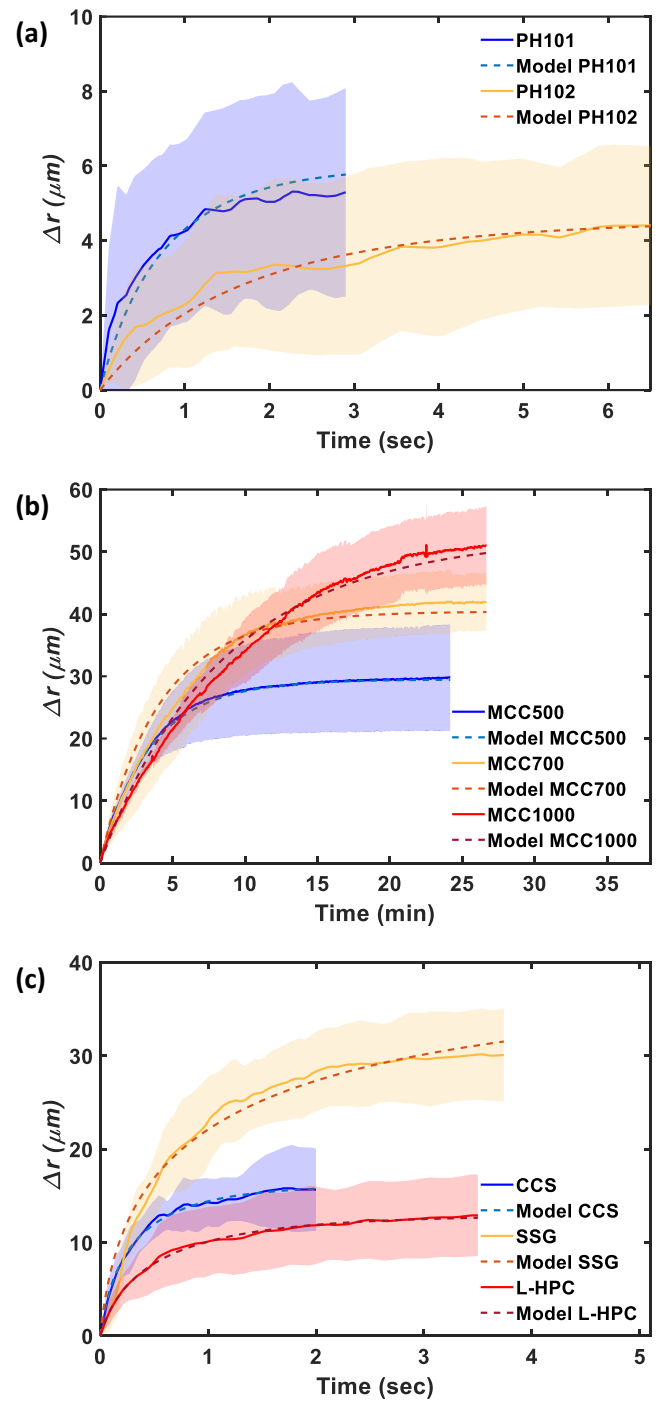

Figure 6: The measured change in radius, $\Delta r=r_{p}-r_{p, 0}$, during particle swelling compared to the swelling model (Eq. 4). (a) PH101 and PH102. (b) MCC 500, MCC700 and MCC1000. (c) L-HPC, CCS and SSG. The solid line and shaded area correspond to the average and standard deviation, respectively, of six particles. 


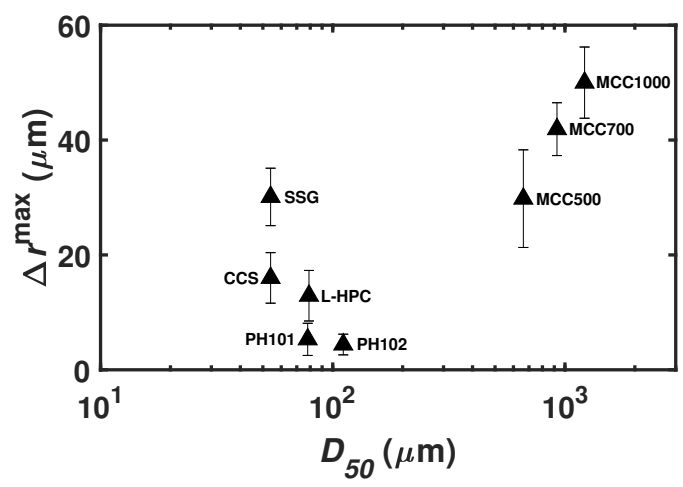

Figure 7: Swelling capacity, $\Delta r^{\max }$, as a function of the particle size, $D_{50}$.

slow initially swelling, the surface water uptake of SSG is low. The hydration of SSG is driven by the interaction between the anionic carboxyl group and water (Zhao and Augsburger, 2005). CCS, however, shows a fast swelling: it swells $14 \mu \mathrm{m}$ in the first $0.8 \mathrm{~s}$ and swells further $2 \mu \mathrm{m}$ within the next $1.6 \mathrm{~s}$. The swelling of CCS is driven by the hydration of the carboxymethyl group (Zarmpi et al., 2017).

\subsubsection{Quantification of Swelling Characteristics}

Characteristic swelling properties were determined for each material to analyse the swelling-controlling mechanisms, i.e. diffusion- or absorption capacity-limited swelling. The swelling model (Eq. 4) was used to extract the diffusion coefficient for all materials and determine the maximum absorption ratio for the disintegrants from the experimental data (Figure 6). For a particle with a small diffusion coefficient, the swelling process was limited by the diffusion of water into a spherical particle. For a particle with a small maximum absorption ratio, the swelling process is limited by the particles liquid absorption capacity.

Considering that only $D$ was fitted, and the other parameters were measured experimentally, the swelling model captures the behaviour of the different MCC grades very well (see RMSE in Table 2). The RMSE is also small for the different disintegrant materials, where both $D$ and $Q^{\max }$ were fitting parameters. The largest RMSE for the disintegrants was observed for the SSG.

$Q^{\max }$ and $D$ of the different materials are given in Table 2. $Q^{\max }$ for the MCC grades is provided in Table 1 as these values were measured following the procedure described in section 2.2.3. The high $Q^{\max }$ value of SSG is attributed to the high spacing, which can be occupied by the water (Rojas et al., 2012; Zarmpi et al., 2017). The $Q^{\max }$ value of CCS and L-HPC are similar as they are both modified cellulose mate- 

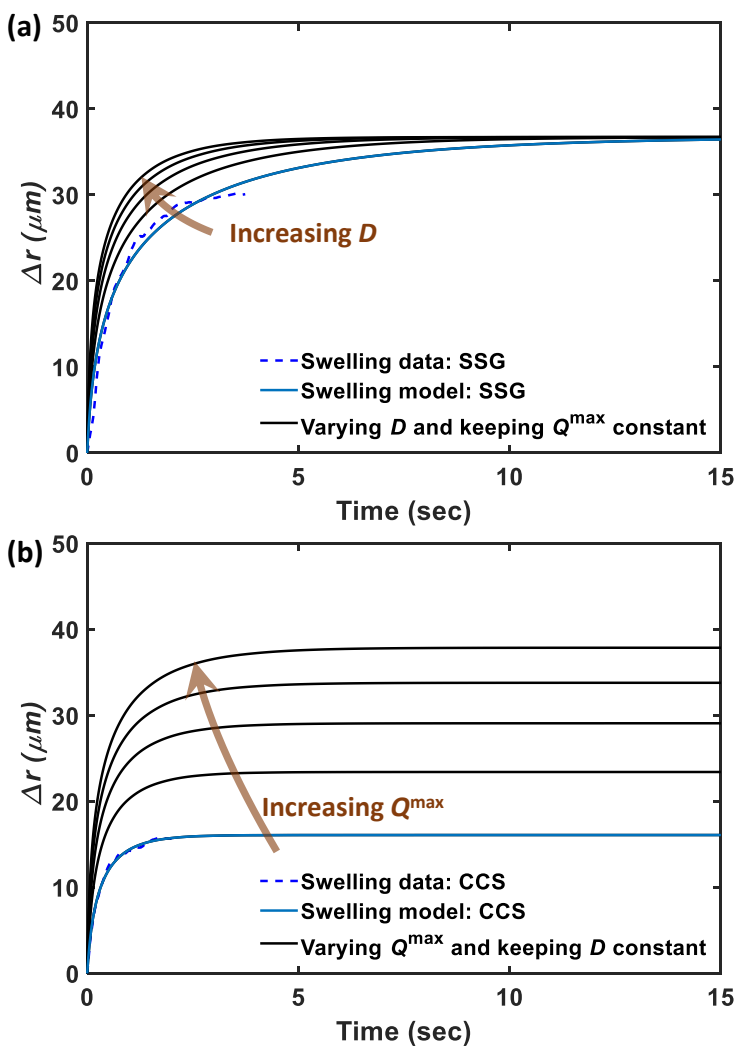

Figure 8: Simulations of swelling profiles using Equation 4 and experimental data (average profiles) of SSG and CCS particles. a) $D$ is varied from $231.14 \mu \mathrm{m}^{2} / \mathrm{s}$ to $739.75 \mu \mathrm{m}^{2} / \mathrm{s}$ with uniform increments of $127.15 \mu \mathrm{m}^{2} / \mathrm{s}$. $Q^{\max }$ is kept constant at $10.04 \mathrm{~g} / \mathrm{g}$. b) $Q^{\max }$ is varied from $3.16 \mathrm{~g} / \mathrm{g}$ to $10.94 \mathrm{~g} / \mathrm{g}$ with uniform increments of $1.72 \mathrm{~g} / \mathrm{g}$. $D$ is kept constant at $739.75 \mu \mathrm{m}^{2} / \mathrm{s}$. 


\begin{tabular}{ccccc}
\hline Material & $Q^{\max }(\mathrm{g} / \mathrm{g})$ & $D\left(\mu m^{2} / \mathrm{s}\right)$ & $\Delta r^{\max }(\mu m)$ & RMSE $(\mu m)$ \\
\hline PH101 & - & 396.39 & 5.3 & 0.35 \\
PH102 & - & 134.68 & 4.4 & 0.38 \\
MCC500 & - & 194.95 & 29.8 & 0.26 \\
MCC700 & - & 279.28 & 41.9 & 1.81 \\
MCC1000 & - & 273.70 & 50.0 & 1.30 \\
CCS & 3.16 & 739.75 & 16.0 & 0.25 \\
SSG & 10.04 & 231.14 & 30.1 & 1.28 \\
L-HPC & 3.19 & 392.61 & 12.9 & 0.18 \\
\hline
\end{tabular}

Table 2: Characteristic swelling properties (maximum absorption ratio, $Q^{\text {max }}$; diffusion coefficient, $D$; swelling capacity, $\Delta r^{\max }$ ) extracted from the experimental data (Figure 6) and the swelling model (Eq. 4). Root mean squared error (RMSE) was calculated to assess the accuracy of the fit between the swelling model and experimental data.

rials. The $Q^{\max }$ values of the different MCC grades are relatively close to each other. The results overall indicate that $Q^{\max }$ primarily depends on the material characteristics and is not influenced by the particle size/shape. The diffusion coefficient, $D$ is higher for CCS due to its fast hydration characteristics (Zarmpi et al., 2017). There is a significant difference between $D$ of PH101 and PH102, where both the size and the moisture content can affect the diffusivity of water in the particle.

The diffusion coefficient of SSG is significantly smaller than that of the other disintegrants, while it has the highest absorption capacity. This means that the swelling process of SSG is limited by the diffusion of liquid into the particle. CCS has a relatively high diffusion coefficient, which results in a faster initial swelling. Since the absorption capacity of CCS is relatively small compared to SSG, the swelling process of CCS is limited by its liquid absorption capacity. The absorption capacity of L-HPC is similar to the one of CCS, but its diffusion coefficient is between the values of SSG and CCS. L-HPC is thus categorised as diffusion and absorption capacitylimited swelling.

Simulations using Eq. 4 (Figure 8) were conducted to gain a better understanding of the effect of $D$ and $Q^{\max }$ on the swelling profile. An increase in $D$, while keeping $Q^{\max }$ constant, accelerates the particle swelling process (Figure 8a). The diffusion coefficient thus primarily affects the swelling rate and not the swelling capacity $\left(\Delta r^{\max }\right)$. On the contrary, increasing $Q^{\max }$ leads to an increase in the swelling capacity and does not affect the initial swelling rate (see Figure $8 \mathrm{~b}$ ).

The swelling capacity increases with increasing particle size for the MCC5001000 particles (Figure 7). This is attributed to the fact that these MCC grades are 
agglomerates of smaller MCC particles and the MCC content therefore increases with size. This also results in a much larger swelling capacity for the MCC5001000 compared to the PH101 and PH102 grades.

Not only the swelling capacity affects the disintegration of a tablet, but also the initial swelling rate which is driven by the water uptake rate. Considering that the majority of pores in a tablet are $<10 \mu \mathrm{m}$ (Ridgway et al., 2017; Markl et al., 2017a, 2018a), the particles will induce stress on the interparticle bonds at an early stage in the swelling process. The initial swelling rate driven by the uptake of liquid on the particle surface might thus be the rate-determining process for tablet disintegration. The particle swelling needed to break up the tablet depends on both the pore size and the strength of the interparticle bonds.

The results indicate that SSG has the largest $Q^{\max }$ but smallest $D$ of the three disintegrants studied. When comparing the results of single particles to the swelling behaviour of a powder compact, the exact formulation and microstructure of a tablet needs to be considered. Quodbach et al. (2014) measured how the disintegration time of dibasic calcium phosphate tablet is affected by the used disintegrant and Botzolakisi and Augsburger (1988) measured the swelling and liquid uptake rate of pure disintegrant compacts. Both indicated that the use of CCS results in faster tablet disintegration compared to a SSG tablet. Considering that the average pore size of tablets are $<10 \mu \mathrm{m}$ (Ridgway et al., 2017; Markl et al., 2017a, 2018a), most interparticle bonds will be interrupted before the particles reach their maximum swelling capacity. The initial particle swelling, and not the swelling capacity of the particles, thus may drive the disintegration process. CCS particles have a higher initial swelling rate compared to SSG tablets even though the SSG swelling capacity is larger. This higher initial swelling rate can thus result in a faster disintegration of a tablet. However, the prediction of the swelling and disintegration behaviour of a powder compact from single particle information requires a better understanding of the interplay between the particle properties, tablet microstructure, the liquid uptake dynamics and the interparticle bonding.

\subsection{Anisotropic Swelling}

The microscope images in Figure 4 indicate that the swelling process is not isotropic for all particles. This is particularly obvious for PH101, where the particle shape changes from a needle- to bottle-like shape during swelling. Such an anisotropic swelling is also reflected in facet-dependent characteristic particle swelling properties. $D, Q^{\max }$ and $\Delta r^{\max }$ were thus estimated along the minor $\left(r_{p \text {,minor }}\right)$ and major $\left(r_{p, \text { major }}\right)$ axes for the non-spherical $\left(S_{50}<0.9\right)$ particles (Figure 9). 

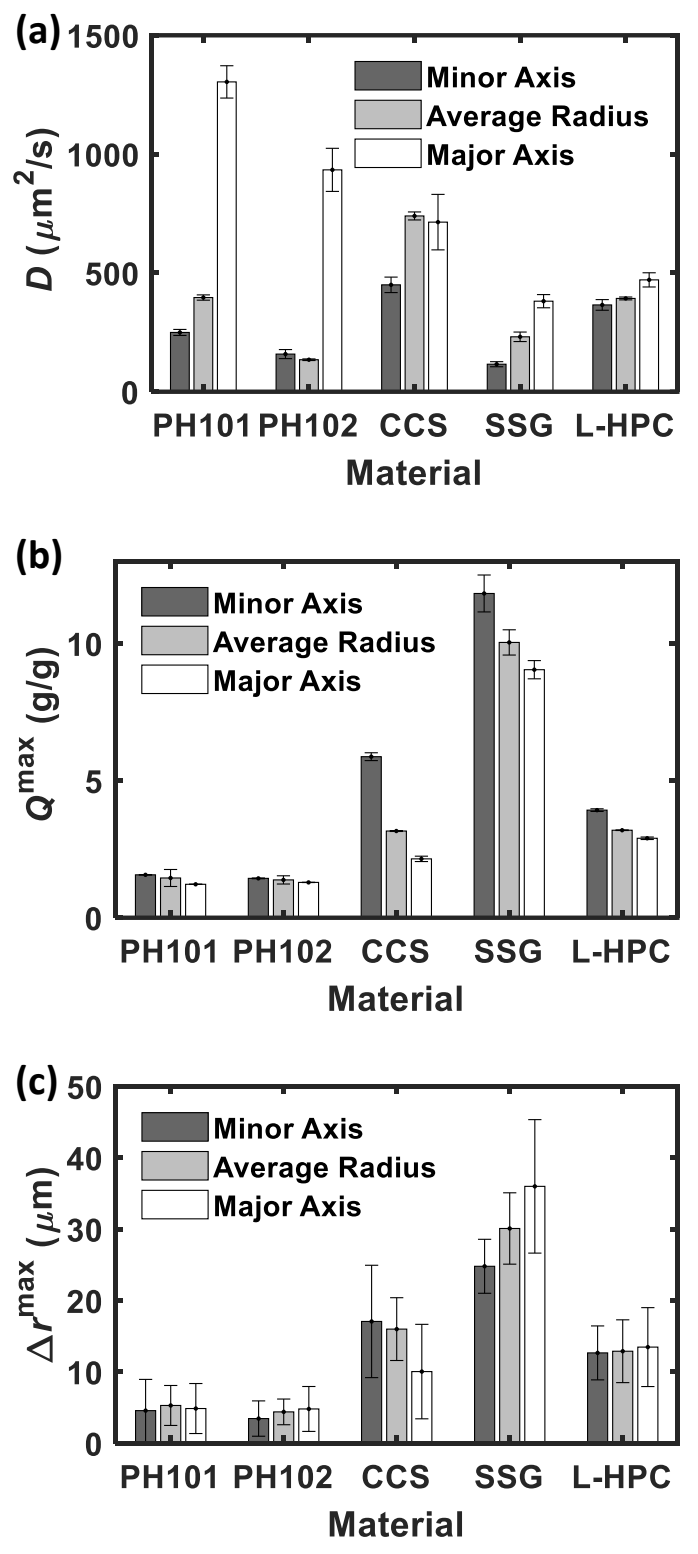

Figure 9: Anisotropic swelling analysis for PH101/PH102 and the three disintegrants. The a) diffusion coefficient, $D$, b) maximum absorption ratio, $Q^{\max }$, and c) swelling capacity, $\Delta r^{\text {max }}$, were extracted for the swelling using the average particle radius, $r_{p}$, the semi-minor axis, $r_{p \text {,minor }}$, and the semi-major axis, $r_{p \text {,major }}$, for each material. A $95 \%$ confidence interval for the fitted parameters, $D$ and $Q^{\max }$, is also shown. 
In the majority of cases, $D, Q^{\max }$ and $\Delta r^{\max }$ values of $r_{p}$ lay between that of $r_{p \text {,minor }}$ and $r_{p \text {,major. }}$ The diffusion of the water into the particles is generally faster along its larger axis. In contrast, the maximum absorption ratio is larger for the smaller particle axis for the disintegrants, while it does not change for PH101 and PH102 particles. In terms of swelling capacity, there is not a noticeable trend across the materials studied. The swelling capacity of SSG is clearly larger along the major axis, while it decreases for CCS from the minor to the major axis. The difference in terms of swelling capacity between the minor and major axes is not significant for PH101, PH102 and L-HPC.

The anisotropic swelling behaviour of PH101, PH102, CCS, SSG and L-HPC is attributed to its fibrous structure (Desai et al., 2015; Thoorens et al., 2014; Sunada and Bi, 2002; Krishnamoorthy et al., 2013). Fibrous materials mainly swell anistropical (Preston and Nimkar, 1949) as they prefer to orientate their linear macromolecules parallel to the fibre axis. The more aligned the macromolecules are, the more these materials swell anistropically.

\section{Conclusion}

The in-situ monitoring of the expansion of single particles paired with a model facilitated the quantification of the swelling of eight pharmaceutical materials. The results clearly highlighted the different swelling behaviour for the various materials, where the swelling capacity (maximum swelling of particle) of the disintegrants follows SSG > CCS > L-HPC and MCC1000 > MCC700 > MCC500 > PH101 > PH102 for the MCC grades. CCS has the highest diffusion coefficient with $D=739.70 \mu \mathrm{m}^{2} / \mathrm{s}$ due to high initial hydration and SSG has the highest maximum absorption ratio with $Q^{\max }=10.04 \mathrm{~g} / \mathrm{g}$ due to high spacing between the cross-linking. In summary for the disintegrants, the swelling performance of SSG is liquid uptake ability limited, whereas it is absorption capacity limited for CCS and L-HPC. The anisotropic swelling is significant for PH101 and PH102 in terms of its diffision of liquid ability with a relative change of $423 \%$ and $457 \%$ for $D$ from the minor to major particle axes for PH101 and PH102, respectively. The absorption capacity considerably depends on the particle facet for CCS, where the relative change of $Q^{\max }$ is $-45 \%$ from the minor to major particle axes. The swelling of single XPVP particles were also studied. However, the results did not reveal any swelling exceeding the resolution limit of the used experimental setup. XPVP in a tablet is described as a strain recovery process (Desai et al., 2012; Quodbach and Kleinebudde, 2014), which is primarily controlled by the compaction process.

Considering the large concentration of MCC in a typical formulation, MCC contributes significantly to tablet swelling and the disintegration process. It is there- 
fore crucial to understand the swelling characteristics of all excipients to predict the swelling of a tablet and hence be able to rank different formulations in terms of disintegration performance.

Quantifying the swelling characteristics of single particles will contribute to a better rational design of a formulation for oral solid dosage forms. It is particularly critical for modelling the tablet disintegration process, where particle swelling is a key performance-controlling mechanism.

In terms of future work, the method presented in this study can be used to measure the swelling of a single particle at different temperatures and dissolution media (e.g. biorelevant media) to better understand the impact of the liquid on the swelling process. Data of single particle swelling at various relevant fluids and temperatures can improve the understanding of variations in the disintegration time in response to liquid temperature changes (Basaleh et al., 2020) and other fluid characteristics.

Furthermore, coupling a swelling model of the materials used in a formulation with liquid transport models (Markl et al., 2017b) will enable the development of a disintegration model. Such a product model can be used to inform formulators to make rational decisions about the formulation and the manufacturing settings.

\section{Acknowledgements}

This work is part funded by the Scottish Research Partnership in Engineering (SRPe): National Manufacturing Institute Scotland - Industry Doctorate Programme (NMIS-IDP) (project award number NMIS-IDP/005). The authors would like to thank the Scottish Research Partnership in Engineering (www.srpe.ac.uk), the Scottish Funding Council (www.sfc.ac.uk) and the National Manufacturing Institute Scotland (www.nmis.scot) for their support in this work. In addition, the authors would like to thank the Future Continuous Manufacturing and Advanced Crystallisation Research Hub (Grant Ref: EP/P006965/1) and the Royal Society (Grant Ref: RSG/R2/180276) for funding this work. The authors would like to acknowledge that this work was carried out in the CMAC National Facility supported by UKRPIF (UK Research Partnership Fund) award from the Higher Education Funding Council for England (HEFCE) (Grant ref HH13054).

\section{References}

Al-Sharabi, M., Markl, D., Mudley, T., Bawuah, P., Karttunen, A.P., Ridgway, C., Gane, P., Ketolainen, J., Peiponen, K.E., Rades, T., Zeitler, J.A., 2020. Simultaneous investigation of the liquid transport and swelling performance during tablet disintegration. International Journal of Pharmaceutics 584, 119380. 
Basaleh, S., Bisharat, L., Cespi, M., Berardi, A., 2020. Temperature: An overlooked factor in tablet disintegration. European Journal of Pharmaceutical Sciences 151, 105388.

Bell, C.L., Peppas, N.A., 1996. An apparatus to measure polymer swelling under load. International Journal of Pharmaceutics 134, 167-172.

Berardi, A., Bisharat, L., Blaibleh, A., Pavoni, L., Cespi, M., 2018. A simple and inexpensive image analysis technique to study the effect of disintegrants concentration and diluents type on disintegration. Journal of Pharmaceutical Sciences 107, 1-10.

Boncelet, C., 2009. Image noise models, in: Bovik, A. (Ed.), The Essential Guide to Image Processing second edition. Academic Press; Pap/Cdr edition, USA. chapter 7, pp. 143-167.

Botzolakisi, J.E., Augsburger, L.L., 1988. Disintegrating agents in hard gelatin capsules. part ii: Swelling efficiency. Drug Development and Industrial Pharmacy 14, 1235 âĂŞ1248.

Desai, P.M., Liew, C.V., Heng, P.W.S., 2015. Review of disintegrants and the disintegration phenomena. Journal of Pharmaceutical Sciences 105, 1-11.

Desai, P.M., Valeri, C., Heng, L.W.S., 2012. Understanding disintegrant action by visualization. Journal of pharmaceutical sciences 101, 2155-2164.

Esteves, L.P., 2011. Superabsorbent polymers: On their interaction with water and pore fluid. Cement \& Concrete Composites 33, 717-724.

Faroongsarng, D., Peck, G.E., 1994. The swelling \& water uptake of tablets iii: Moisture sorption behavior of tablet disintegrants. Drug Development and Industrial Pharmacy 20, 779-798.

Gasmi, H., F. Danede, J.S., Siepmann, F., 2015. Does plga microparticle swelling control drug release? new insight based on single particle swelling studies. Journal of Controlled Release 213, 120-127.

Huyghe, L.M., Janssen, J.D., 1997. Quaadrif hasic mechanics of swelling incompressible porous media. International Journal of Engineering Science 35, 793-80.

Jenkins, L.M., Donald, A.M., 1997. Use of the environmental scanning electron microscope for the observation of the swelling behaviour of cellulosic fibres. Scanning 19, 92-97. 
Kimber, J., Kazarian, S.G., Stepanek, F., 2012. Modelling of pharmaceutical tablet swelling and dissolution using discrete element method. Chemical Engineering Science 69, 394-403.

Kleinebudde, P., Knop, K., 2007. Direct pelletization of pharmaceutical pellets in fluid-bed processes, in: Salma, A.D., Hounslow, M.J., Seville, J.P.K. (Eds.), Handbook of powder technology: Granulation. Elsevier. chapter 17, pp. 779-811.

Krishnamoorthy, V., Prasad, V.P.R., Sen, S., 2013. Clozapine-sodium starch glycolate dispersions: in vitro dissolution behaviour, physicochemical characterisation and release kinetic model fitting. Malaysian Journal of Pharmaceutical Sciences 11, 49-70.

Manjón, J.V., Carbonell-Caballero, J., Lull, J.J., García-Martí, G., MartíÄś-Bonmatí, L., Robles, M., 2008. Mri denoising using non-local means. Medical image analysis $12,514-23$.

Markl, D., Strobel, A., Schlossnikl, R., Bøtker, J., Bawuah, P., Ridgway, C., Rantanen, J., Rades, T., Gane, P., Peiponen, K.E., Zeitler, J.A., 2018a. Characterisation of pore structures of pharmaceutical tablets: A review. International Journal of Pharmaceutics 538, 188-214.

Markl, D., Wang, P., Ridgway, C., Karttunen, A.P., Bawuah, P., Ketolainen, J., Gane, P., Peiponen, K.E., Zeitler, J.A., 2018b. Resolving the rapid water absorption of porous functionalised calcium carbonate powder compacts by terahertz pulsed imaging. Chemical Engineering Research and Design 132, 1082 - 1090.

Markl, D., Wang, P., Ridgway, C., Karttunen, A.P., Chakraborty, M., Bawuah, P., Pääkkönen, P., Gane, P., Ketolainen, J., Peiponen, K.E., Zeitler, J.A., 2017a. Characterization of the Pore Structure of Functionalized Calcium Carbonate Tablets by Terahertz Time-Domain Spectroscopy and X-Ray Computed Microtomography. Journal of Pharmaceutical Sciences 106, 1586-1595.

Markl, D., Yassin, S., Wilson, D.I., Goodwin, D.J., Anderson, A., Zeitler, J.A., 2017b. Mathematical modelling of liquid transport in swelling pharmaceutical immediate release tablets. International Journal of Pharmaceutics 526, 1-10.

Markl, D., Zeitler, J.A., 2017. A review of disintegration mechanisms and measurement techniques. Pharmaceutical Research 34, 890-917.

Omidian, H., Hashemi, S.A., Sammes, P.G., Meldrum, I., 1998. A model for the swelling of superabsorbent polymers. Polymer 39, 6697-6704. 
Patel, N.R., Hopponent, R.E., 1966. Mechanism of action of starch as a disintegrating agent in aspirin tablets. Journal of Pharmaceutical Sciences 55, 1065-1068.

Perona, P., Malik, J., 1990. Scale space and edge detection using anisotropic diffusion. Transactions on Pattern Analysis and Machine Intelligence 12, 629-639.

Preston, J.M., Nimkar, M.V., 1949. Measuring the swelling of fibres in water. Journal of the Textile Institute Proceedings 40, 674-688.

Quodbach, J., Kleinebudde, P., 2014. Systematic classification of tablet disintegrants by water uptake and force development kinetics. Journal of Pharmacy and Pharmacology 66, 1429-1438.

Quodbach, J., Kleinebudde, P., 2015. A critical review on tablet disintegration. Pharmaceutical Development and Technology 21, 1-12.

Quodbach, J., Moussavi, A., Tammer, R., Frahm, J., Kleinebudde, P., 2014. Assessment of disintegrant efficacy with fractal dimensions from real-time mri. International Journal of Pharmaceutics 475, 605-612.

Reier, G.E., Shangraw, R.F., 1966. Microcrystalline cellulose in tableting. Journal of Pharmaceutical Sciences 55, 510-514.

Ridgway, C., Bawuah, P., Markl, D., Zeitler, J.A., Ketolainen, J., Peiponen, K.E., Gane, P., 2017. On the role of API in determining porosity, pore structure and bulk modulus of the skeletal material in pharmaceutical tablets formed with MCC as sole excipient. International Journal of Pharmaceutics 526, 321-331.

Rojas, J., Guisao, S., Ruge, V., 2012. Functional assessment of four types of disintegrants and their effect on the spironolactone release properties. Journal of the American Association of Pharmaceutical Scientists 13, 1054-1062.

Rudnic, E., Rhodes, C., Welch, S., Bernard, P., 1982. Evaluations of the mechanism of disintegrant action. Drug Development and industrial pharmacy 8, 87-109.

Russ, J.C., Neal, F.B., 2016. The Image Processing Handbook, Seventh edition. CRC Press LLC.

Russell, A., Šibanc, R., Dreu, R., Müller, P., 2018. Mechanics of pharmaceutical pellets- constitutive properties, deformation, and breakage behavior. Journal of Pharmaceutics Sciences 107, 571-586. 
Schott, H., 1992. Kinetics of swelling of polymers and their gels. Journal of Pharmaceutical Sciences 81, 467-470.

Shah, U., Augsburger, L., 2002. Multiple sources of sodium starch glycolate, nf: Evaluation of functional equivalence and development of standard performance tests. Pharmaceutical Development and Technology 7, 345-359.

Sunada, H., Bi, Y., 2002. Preparation, evaluation and optimization of rapidly disintegrating tablets. Powder Technology 122, 188-198.

Sweijen, T., Chareyre, B., Hassanizadeh, S., Karadimitriou, N., 2017a. Grain-scale modelling of swelling granular materials; application to super absorbent polymers. Powder Technology 318, 411-422.

Sweijen, T., van Duijna, C.J., Hassanizadeh, S.M., 2017b. A model for diffusion of water into a swelling particle with a free boundary: Application to a super absorbent polymer particle. Chemical Engineering Science 172, 407-413.

Thoorens, G., Krier, F., Leclercq, B., Carlin, B., Evrard, B., 2014. Microcrystalline cellulose, a direct compression binder in a quality by design environmentâĂ $\breve{T a}$ review. International Journal of Pharmaceutics 473, 64-72.

York, P., 2018. Design of dosage forms, in: Aulton, M.E., Taylor, K.M.G. (Eds.), Aulton's Pharmaceutics The Design and Manufacture of Medicines, fift edition. Elsevier. chapter 1, pp. 6-17.

Zarmpi, P., Flanagan, T., Meehan, E., Mann, J., Fotaki, N., 2017. Biopharmaceutical aspects and implications of excipient variability in drug product performance. European Journal of Pharmaceutics and Biopharmaceutics 111, 1-17.

Zhao, N., Augsburger, L.L., 2005. The influence of swelling capacity of superdisintegrants in different ph media on the dissolution of hydrochlorothiazide from directly compressed tablets. American Association of Pharmaceutical Scientists $6,120-126$. 